\title{
Incidental Serous Tubal Intraepithelial Carcinoma Detected by a Surgery for Ectopic Pregnancy
}

\author{
Takuro Yamamoto*, Koki Shimura, Takuya Sugahara, Nozomi Ogiso, Tomoharu Okubo \\ Department of Obstetrics and Gynecology, Japanese Red Cross Kyoto Daiichi Hospital, Honmachi, Higashiyama-Ku, \\ Kyoto, Japan \\ Email: *teku@koto.kpu-m.ac.jp
}

How to cite this paper: Yamamoto, T., Shimura, K., Sugahara, T., Ogiso, N. and Okubo, T. (2020) Incidental Serous Tubal Intraepithelial Carcinoma Detected by a Surgery for Ectopic Pregnancy. Open Journal of Obstetrics and Gynecology, 10, 738-743. https://doi.org/10.4236/ojog.2020.1050068

Received: April 24, 2020

Accepted: May 15, 2020

Published: May 18, 2020

Copyright () 2020 by author(s) and Scientific Research Publishing Inc. This work is licensed under the Creative Commons Attribution International License (CC BY 4.0).

http://creativecommons.org/licenses/by/4.0/

\section{Open Access}

\begin{abstract}
Serous tubal intraepithelial carcinoma is a putative precursor of high-grade serous carcinoma, which is the most common histological type of ovarian or pelvic peritoneal cancer. Serous tubal intraepithelial carcinoma is commonly found in patients with breast cancer susceptibility gene mutations who undergo risk-reducing salpingo-oophorectomy. Incidental serous tubal intraepithelial carcinoma found by a non-prophylactic surgery is rare. A 33-year-old woman referred to our hospital for a diagnosis of ectopic pregnancy. She underwent a laparoscopic right salpingectomy. Pathologically, ectopic pregnancy in the ampulla of the right fallopian tube was confirmed and serous tubal intraepithelial carcinoma was observed in the fallopian tube. Subsequently, she underwent a laparoscopic hysterectomy, bilateral oophorectomy, and left salpingectomy as additional treatment. She has experienced no recurrence thus far for 37 months since the surgery.
\end{abstract}

\section{Keywords}

Serous Tubal Intraepithelial Carcinoma, Ectopic Pregnancy, Laparoscopic Surgery

\section{Introduction}

Serous tubal intraepithelial carcinoma (STIC) is a putative precursor of high-grade serous carcinoma (HGSC), which is the most common histological type of ovarian or pelvic peritoneal cancer. STIC is commonly found in patients with breast cancer susceptibility gene (BRCA) mutations who undergo risk-reducing salpingo-oophorectomy (RRSO). Incidental STIC found by a non-prophylactic surgery is rare. Here, we present a case of STIC incidentally found by a surgery 
for an ectopic pregnancy.

\section{Case Presentation}

A 33-year-old woman, gravida 7 para 2 (5 artificial abortions and 2 vaginal labors), presented to a hospital complaining of irregular genital bleeding and lower abdominal pain, with a positive gestational test. She was suspected of having an ectopic pregnancy. She referred to our hospital for a diagnosis and surgery. She was in 5 weeks and 5 days of gestation, based on the date of her last menstrual period. She had no family history of cancer. Upon the first clinical examination, genital bleeding and lower abdominal tenderness with peritoneal irritation were present. Transvaginal sonography revealed a moderate to large intra-abdominal hemorrhage in the Douglas' pouch and a cystic region that appeared to be the gestational sac in the right fallopian tube. The results of biochemical blood examination were almost within normal limits, except for a lower hemoglobin concentration and elevated $\beta$ human chorionic gonadotropin $(1209.4 \mathrm{mIU} / \mathrm{mL})$. Accordingly, she was diagnosed of having a right fallopian tube rupture due to ectopic pregnancy, and a laparoscopic right salpingectomy was performed. There were no significant intraoperative findings, except for the ectopic pregnancy. She was pathologically diagnosed with ectopic pregnancy at the ampulla of the right fallopian tube and STIC. We suggested either follow-up or additional treatment to the patient. She and her husband decided to undergo a total laparoscopic hysterectomy and bilateral salpingo-oophorectomy. During the surgery, cytology results of the peritoneal washings were negative for malignancy. There was no residual tumor pathologically in the uterus or adnexa. She has been followed up for recurrence by examining level of serum CA125 and using computed tomography. She received estrogen replacement therapy using estradiol transdermal gel ( $1 \mathrm{mg} /$ day). At the time of this report, she has no evidence of disease 37 months after the surgery.

\section{Pathological Findings}

A rupture of the ampulla of the right fallopian tube was observed. Villi were present in the fallopian tube. In addition, epithelial tube thickness was partially increased. There was increased epithelial cell stratification, enlarged nuclei and nuclear rounding, and hyperchromasia (Figure 1(a)). Immunohistochemical staining was highly positive for MIB1 and p53 (Figure 1(b) and Figure 1(c), respectively). The patient was diagnosed with ectopic pregnancy and STIC in the right fallopian tube.

\section{Discussion}

To our knowledge, there is no previous report of STIC discovered along with ectopic pregnancy. STIC may be precursor lesions of HGSC of the ovary or fallopian tube. RRSO in carriers of BRCA mutations or women with high risk of heredity breast and ovarian carcinoma (HBOC) is a choice to prevent ovarian 

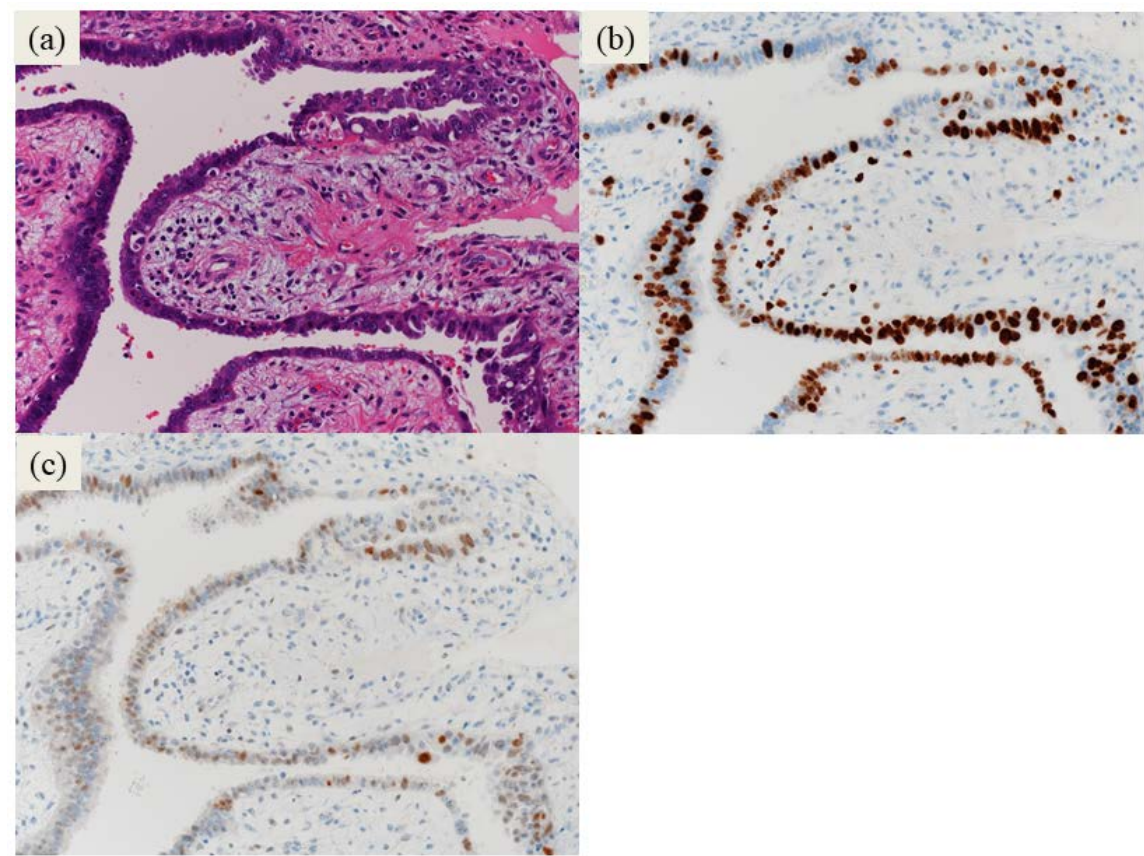

Figure 1. Microscopic findings of serous tubal intraepithelial carcinoma (STIC) in the fallopian tubal epithelium. (a) Hematoxylin and eosin staining. The STIC lesion stained (b) highly positive for MIB-1, and (c) p53.

and tubal cancers [1] [2]. Incidence of STIC was reported to be $0.6 \%-6.0 \%$ in patients with BRCA1 or BRCA2 mutations who undergo RRSO and $1.0 \%-1.6 \%$ in patients with strong family history [3]-[8]. Upon reviewing literature, STIC was detected in 27 of 952 cases (2.8\%) of patients with BRCA mutations and 5 of 389 cases $(1.3 \%)$ of patients with a family history of ovarian or breast cancer [3]-[8]. Among the patients with BRCA mutations or strong family histories who have undergone RRSO, the mean age at diagnosis of STIC was $53.0-54.3$ years (range 39 - 77) [3] [4]. In our case, although she was not examined BRCA mutation status, she did not have a family history of ovarian or breast cancer. She was accidentally diagnosed as STIC at 33 years old.

There are no common criteria for absolute diagnosis of STIC. Currently, most STICs have been diagnosed using a combination of morphology and immunohistochemical analysis for p53 and Ki-67. Morphologic features of STIC include at least 1 mitotic figure, epithelial stratification (more than 2 cell layers), apoptotic bodies, nuclear enlargement and/or nuclear rounding, marked pleomorphism, abnormal chromatin and nuclear molding [9]. TP53 mutation is assumed when positive immunohistochemical staining for p53 is noted for more than $75 \%$ of cells or completely negative. Using a Ki-67 labeling index threshold of $10 \%$ to differentiate between STIC and normal fallopian tube epithelium, the sensitivity and specificity were $100 \%$ and $96.4 \%$, respectively [10]. MIB-1 and Ki-67 labeling were used to diagnose this case. We diagnosed this case as STIC by the pathological findings of the morphology, TP53 mutation, and high Ki-67 labeling index. 
There have been few reports of incidental STIC in patients unknown to carry BRCA mutations. In women with low risk of HBOC, the incidence of STIC was 4 in 522 cases (0.77\%) [11]. Upon reviewing the literature, the median age at the time of diagnosis of incidental STIC by a surgery for benign disease was 61 years (range, 39 - 86 years), and only one of 44 cases was diagnosed among patients younger than 40 years old, while 5 of 44 cases were diagnosed in patients younger than 50 years old [11] [12] [13] [14]. The surgeries used to treat those patients included 35 bilateral salpingo-oophorectomies, 5 unilateral salpingo-oophorectomies, and 5 salpingectomies, with one case of both unilateral salpingo-oophorectomy and contralateral salpingectomy. Unilateral or bilateral adnexa persisted without additional treatment in only 4 of 44 cases [11] [12] [13] [14]. The conditions that were comorbid with STIC included 10 case of endometriosis, 5 ovarian serous borderline tumors, 4 ovarian serous benign tumors, 4 endometrioid endometrial cancers, 3 leiomyomas, 2 mature cystic teratomas, 2 endometrial hyperplasia, 2 non-gynecological cancers, 1 ovarian mucinous benign tumor, and 1 ovarian fibroma [11] [12] [13] [14]. Additional surgeries were performed in 6 patients with pure STIC at primary diagnosis, and 3 of those tumors were upstaged to HGSC [11] [12] [13] [14]. Wethington et al. reported that among cases of isolated STIC after RRSO, the benefit of surgical staging is minimal, and short-term (range, 16 - 44 months) clinical outcomes are favorable [4]. However, in our case, the patient underwent only a right salpingectomy. Very few cases of STIC treated without oophorectomy have been reported. It is still unclear whether an additional surgery is necessary in such cases. We provided the patient with enough available information, and she decided to undergo a total laparoscopic hysterectomy, left salpingectomy and bilateral oophorectomy. Fortunately, there was no residual tumor. However, she was in her 30s, and estrogen replacement therapy was needed.

In conclusion, this is the first report of STIC diagnosed in a patient with an ectopic pregnancy in her early 30s. STIC might cause dysfunction of the ciliated epithelium in the fallopian tube. Therefore, the fallopian tube of patients with ectopic pregnancy should be closely observed, even for patients with no family history of ovarian or breast cancer.

\section{Consent}

Written informed consent was obtained from the patient for publication of this case report.

\section{Conflicts of Interest}

The authors declare no conflicts of interest regarding the publication of this paper.

\section{References}

[1] Kauff, N.D., Satagopan, J.M., Robson, M.E., Scheuer, L., Hensley, M., Hudis, C.A., Ellis, N.A., Boyd, J., Borgen, P.I., Barakat, R.R., et al. (2002) Risk-Reducing Salpin- 
go-Oophorectomy in Women with a BRCA1 or BRCA2 Mutation. The New England Journal of Medicine, 346, 1609-1615. https://doi.org/10.1056/NEJMoa020119

[2] Meeuwissen, P.A., Seynaeve, C., Brekelmans, C.T., Meijers-Heijboer, H.J., Klijn, J.G. and Burger, C.W. (2005) Outcome of Surveillance and Prophylactic Salpingo-Oophorectomy in Asymptomatic Women at High Risk for Ovarian Cancer. Gynecologic Oncology, 97, 476-482. https://doi.org/10.1016/j.ygyno.2005.01.024

[3] Manchanda, R., Abdelraheim, A., Johnson, M., Rosenthal, A.N., Benjamin, E., Brunell, C., Burnell, M., Side, L., Gessler, S., Saridogan, E., et al. (2011) Outcome of Risk-Reducing Salpingo-Oophorectomy in BRCA Carriers and Women of Unknown Mutation Status. BJOG, 118, 814-824.

https://doi.org/10.1111/j.1471-0528.2011.02920.x

[4] Wethington, S.L., Park, K.J., Soslow, R.A., Kauff, N.D., Brown, C.L., Dao, F., Otegbeye, E., Sonoda, Y., Abu-Rustum, N.R., Barakat, R.R., et al. (2013) Clinical Outcome of Isolated Serous Tubal Intraepithelial Carcinomas (STIC). International Journal of Gynecological Cancer, 23, 1603-1611.

https://doi.org/10.1097/IGC.0b013e3182a80ac8

[5] Carcangiu, M.L., Peissel, B., Pasini, B., Spatti, G., Radice, P. and Manoukian, S. (2006) Incidental Carcinomas in Prophylactic Specimens in BRCA1 and BRCA2 Germ-Line Mutation Carriers, with Emphasis on Fallopian Tube Lesions: Report of 6 Cases and Review of the Literature. The American Journal of Surgical Pathology, 30, 1222-1230. https://doi.org/10.1097/01.pas.0000202161.80739.ac

[6] Powell, C.B., Chen, L.M., McLennan, J., Crawford, B., Zaloudek, C., Rabban, J.T., Moore, D.H. and Ziegler, J. (2011) Risk-Reducing Salpingo-Oophorectomy (RRSO) in BRCA Mutation Carriers: Experience with a Consecutive Series of 111 Patients Using a Standardized Surgical-Pathological Protocol. International Journal of Gynecological Cancer, 21, 846-851. https://doi.org/10.1097/IGC.0b013e31821bc7e3

[7] Callahan, M.J., Crum, C.P., Medeiros, F., Kindelberger, D.W., Elvin, J.A., Garber, J.E., Feltmate, C.M., Berkowitz, R.S. and Muto, M.G. (2007) Primary Fallopian Tube Malignancies in BRCA-Positive Women Undergoing Surgery for Ovarian Cancer Risk Reduction. Journal of Clinical Oncology, 25, 3985-3990. https://doi.org/10.1200/JCO.2007.12.2622

[8] Finch, A., Shaw, P., Rosen, B., Murphy, J., Narod, S.A. and Colgan, T.J. (2006) Clinical and Pathologic Findings of Prophylactic Salpingo-Oophorectomies in 159 BRCA1 and BRCA2 Carriers. Gynecologic Oncology, 100, 58-64.

https://doi.org/10.1016/j.ygyno.2005.06.065

[9] Visvanathan, K., Vang, R., Shaw, P., Gross, A., Soslow, R., Parkash, V., Shih Ie, M. and Kurman, R.J. (2011) Diagnosis of Serous Tubal Intraepithelial Carcinoma Based on Morphologic and Immunohistochemical Features: A Reproducibility Study. The American Journal of Surgical Pathology, 35, 1766-1775. https://doi.org/10.1097/PAS.0b013e31822f58bc

[10] Kuhn, E., Kurman, R.J., Sehdev, A.S. and Shih Ie, M. (2012) Ki-67 Labeling Index as an Adjunct in the Diagnosis of Serous Tubal Intraepithelial Carcinoma. International Journal of Gynecological Pathology, 31, 416-422. https://doi.org/10.1097/PGP.0b013e31824cbeb4

[11] Rabban, J.T., Garg, K., Crawford, B., Chen, L.M. and Zaloudek, C.J. (2014) Early Detection of High-Grade Tubal Serous Carcinoma in Women at Low Risk for Hereditary Breast and Ovarian Cancer Syndrome by Systematic Examination of Fallopian Tubes Incidentally Removed during Benign Surgery. The American Journal of Surgical Pathology, 38, 729-742. https://doi.org/10.1097/PAS.0000000000000199

[12] Chay, W.Y., McCluggage, W.G., Lee, C.H., Kobel, M., Irving, J., Millar, J., Gilks, 
C.B. and Tinker, A.V. (2016) Outcomes of Incidental Fallopian Tube High-Grade Serous Carcinoma and Serous Tubal Intraepithelial Carcinoma in Women at Low Risk of Hereditary Breast and Ovarian Cancer. International Journal of Gynecological Cancer, 26, 431-436. https://doi.org/10.1097/IGC.0000000000000639

[13] Morrison, J.C., Blanco, L.Z., Vang, R. and Ronnett, B.M. (2015) Incidental Serous Tubal Intraepithelial Carcinoma and Early Invasive Serous Carcinoma in the Nonprophylactic Setting: Analysis of a Case Series. The American Journal of Surgical Pathology, 39, 442-453. https://doi.org/10.1097/PAS.0000000000000352

[14] Vaughan, M.H., Modesitt, S.C., Mo, Y. and Trowbridge, E.R. (2015) Serous Tubal Intraepithelial Carcinoma: An Incidental Finding at the Time of Prophylactic Bilateral Salpingo-Oophorectomy. Case Reports in Obstetrics and Gynecology, 2015, Article ID: 760429. https://doi.org/10.1155/2015/760429 